\title{
Hartman's solution or normal saline in the treatment of hyperemesis gravidarum among South East Asian population: a randomised controlled trial”
}

Adibah Ia , Khursiah $D^{\mathrm{b}}$, Ahmad Alc , and Zaki NNM ${ }^{\mathrm{d}}$

a Senior lecturer and Specialist, b Medical officer, c Senior lecturer and Specialist, and ${ }^{\mathrm{d}}$ Head of Department, Department of Obstetrics and Gynaecology, School of Medical Sciences, Science University of Malaysia, 16150 Kubang Kerian, Kelantan Malaysia

\section{ABSTRACT}

Introduction: The aim of treatment for hyperemesis gravidarum is to stop vomiting, correction of dehydration, starvation and electrolytes imbalance. The common types of fluid used for fluid replacement are isotonic solutions like normal saline and hartman's solutions. The absence of potassium in normal saline makes hartman's solution superior but there is a possibility that the lactate component in hartman's solution could worsen the starvation state of the patients. This study is to evaluate which of these two solutions is more effective for fluid replacement in hyperemesis gravidarum. The objectives are to compare which solution corrects dehydration, hypokalaemia and acetonuria faster and to evaluate whether the ketosis state is aggravated by lactate component in hartman's solution. Materials and Methods: Patients with hyperemesis gravidarum were randomised to receive either Hartman;'s solution or normal saline at the rate of $125 \mathrm{mls} /$ hour. Blood urea and serum electrolytes, haematocrit, lactate and urine acetone were taken during admission and repeated every 12 hours. The volume of fluid required to correct dehydration, hypokalaemia and acetonuria were compared. Comparison of the pre and post treatment level of serum lactate were also done. Results: Both hartman's solution and normal saline are both effective in correcting dehydration (11.52 3.28 pints versus $11.94 \pm 2.30$ pints respectively) and acetonuria (11.64 \pm 2.75 pints versus $11.64 \pm 2.54$ pints respectively). A lower volume of hartman's solution was needed to correct hypokalaemia ( $8.34 \pm 2.44$ pints versus $8.88 \pm 2.63$ pints) but was not statistically significant. Ketonaemia was not made worse after treatment with hartman's solution. Conclusion: Normal saline and hartman's solution are equally effective in treating complications of hyperemesis gravidarum.

KEYWORDS: Hyperemesis Gravidarum, Fluid Replacement, Dehydration, Hypokalaemia, Ketosis

INTRODUCTION

Nausea and vomiting has been associated with early pregnancy from the very beginning of life. It occurs in approximately $56 \%$ of all pregnant women. 1 Hyperemesis gravidarum (HG), which is a severe form of nausea and vomiting in pregnancy, occurs in 3.5 per 1000 pregnancies more common in native Americans, Eskimo, African and Asian communities.1,2,3

Its pathophysiology remains uncertain and there is no universally agreed therapy.1-5 At present, the management of HG include hospitalisation, intravenous fluid and electrolytes replacement, thiamine supplementation, use of conventional anti-emetics and psy-

\section{Correspondence author}

Dr. Adibah Ibrahim M.Med(O\&G)

Senior lecturer and Specialist

Department of Obstetrics and Gynaecology

School of Medical Sciences

Science University of Malaysia

16150 Kubang Kerian, Kelantan

Malaysia

Tel: $+60129089756 /+6097663320$

E-mail: dradibah70@yahoo.com.my chological support.4,5

Various types of fluid are used to correct dehydration in HG. Infusion of dextrose containing fluids is too dangerous and is to be avoided. This is because Wernicke's encephalopathy may be precipitated by carbohydrate rich intravenous fluids. The hyponatraemia demands infusion of sodium containing fluids, and as such Normal saline $(\mathrm{NaCl} 0.9 \% ; 150 \mathrm{mmol} / \mathrm{L}$ sodium) or Hartmann's solution ( $\mathrm{NaCl} 0.6 \% ; 131 \mathrm{mmol} / \mathrm{L}$ sodium) are considered appropriate solutions. 6 The fact that there is absence of potassium supplement in normal saline favours the use of Hartmann's solution. On the other hand there is a possibility that lactate component in Harmann's solution may worsen the ketosis state in patients with HG.

\section{OBJECTIVES}

This study was performed to look into which fluid, either normal saline or hartman's solution is more effective in correcting dehydration, hypokalaemia and ketosis in hyperemesis gravidarum and to evaluate if the ketones in hartman's solution will worsen the ketosis state of these patients. 


\section{METHODOLOGY}

This study was performed in the gynaecology ward of Hospital USM from 31st July 2006 till 1st February 2007. An approval from the ethical committee of USM was obtained prior to the study.

Patients who were admitted to the gynaecology ward of Hospital USM with hyperemesis gravidarum were randomly selected based on block of three. Those with singleton pregnancy and less than 20 weeks pregnant with hyperemesis gravidarum were included into the study.

Patients agreeing to participate into this study were again randomised into 2 groups i.e. group A (receiving hartman's solution) and group B (receiving normal saline) by picking one of two envelopes labelled ' $A$ ' and ' $B$ '. Baseline investigations which consisted of haematocrit level, blood urea and electrolytes (BUSE), serum lactate and urine ketones were taken from all patients and were repeated every 12 hours until normalization of all parameters was achieved. All patients were instructed to fast for the first 24 hours of admission. Anti-emetic metochlorpramide $10 \mathrm{mg}$ was administered intravenously three times a day to all patients. Patients in the normal saline group received 6 pints of normal saline over 24 hours (at the rate of $125 \mathrm{mls} /$ hour), while those in the hartman's solution group received 6 pints of hartmann's solution over 24 hours at the same rate, until dehydration (assessed clinically and based on haematocrit levels and urine specific gravity), ketosis state (assessed from urine ketones and serum lactate level) and hypokalaemic state (assessed from serum potassium level) was corrected.

Patients were allowed to go back home when the vomiting ceased and the dehydration, ketosis and hypokalaemic state were corrected.

The data was collated and analysed using chi- square test from the statistical software SPSS version 14.0.

\section{RESULTS}

To evaluate the effectiveness of hartman's solution and normal saline in HG patients, this study subjected 100 patients to the protocol of either hartman's solution ( $n=50$ patients) or normal saline ( $n=50$ patients). As seen in Table I, there was no significant difference in age $(25.22 \pm 6.06$ years versus $25.36 \pm 4.58$ years $)$, gravidity $(1.70 \pm 1.11$ versus $2.34 \pm 1.57)$, gestational age $(10.70 \pm 3.21$ weeks versus $10.58 \pm 4.12$ weeks $)$ and body mass index (BMI) $(25.37 \pm 2.49 \mathrm{~kg} / \mathrm{m} 2$ versus $26.14 \pm 1.52 \mathrm{~kg} / \mathrm{m} 2$ ) between patients treated with hartman's solution and normal saline. The incidence of HG in this study decreased as gravidity increased (gravida 2, $\mathrm{n}=24(24 \%)$; gravida 3, $\mathrm{n}=4(4 \%)$; gravida 4, $\mathrm{n}=13(13 \%)$; gravida $5, \mathrm{n}=5(5 \%)$, gravida $6, \mathrm{n}=3(3 \%))$.

The hydration state of the patients was assessed clinically as well as assessing the haematocrit level and urine specific gravity level. It required a mean of
$11.73 \pm 2.83$ pints of either fluid to correct dehydration among the patients. As shown in Table II, there was no significant difference in the amount of either hartman's solution or normal saline required to correct dehydration.

All patients recruited into this study had normal level of sodium (mean: $136.5 \pm 1.34 \mathrm{mmol} / \mathrm{L}$ versus 136.1 $\pm 0.79 \mathrm{mmol} / \mathrm{L})$ in both the hartman's solution and normal saline groups. There was no statistical difference between both groups $(p=0.34)$. With regard to potassium level, although lower level of hartmann's solution was required to correct hypokalaemia, the difference not significant (Table III).

The presence of acetonuria is one of the indicators of starvation. As seen in Table IV, there was no difference in the amount of fluid and the time taken to clear acetonuria for both hartman's solution and normal saline group. (11.64 \pm 2.54 pints; $46.56 \pm 10.18$ hours for normal saline group and $11.64 \pm 2.75$ pints; $46.56 \pm 11.01$ hours for hartman's solution) respectively.

In the hartman's solution group, there was no increase in the lactate levels before and after treatment (mean $1.13 \mathrm{mmol} / \mathrm{L}$ and $1.11 \mathrm{mmol} / \mathrm{L}, \mathrm{p}=0.283)$, as seen in Table V.

\section{DISCUSSION}

The exact pathogenesis of HG is poorly understood. However there are a few associations that could increase the incidence of HG. Though the association of HG and oestrogen levels as well as 17- hydroxyprogesterone is not proven, it was observed that the risk of HG increases among patients taking contraceptive pills prior to pregnancy.7,8 In this study the increased incidence of $\mathrm{HG}$ among women with higher BMI may support the role of oestrogen in HG. It was also noted in this study that HG occurs more frequently in nulliparous women in their first pregnancy. This finding is consistent with most of other epidemiological studies of HG.7-10

Many studies have been performed to look into the pharmacological as well as non- pharmacological aspect of treatment to reduce vomiting in HG. A few studies looked into the various treatment used to reduce the risk of thiamine deficiency which might lead to Wernicke's encephalopathy.11-14 However, no study has compared the different fluids used for fluid replacement for HG.14

This study compared the two types of isotonic solutions normally used for fluid replacement in HG. It demonstrated that both fluids are as effective in correcting the dehydration state of the patients as shown in Table I. Though the amount of fluid required for fluid replacement is affected by ongoing loss, it did not seem to affect the result of the study.

Normal saline infusion alone cannot treat the hypokalaemia in patients with HG due to the absence of po- 
tassium. Thus, to correct this condition, additional of potassium ion supplement into the solution is required. Many a times we see infiltration at the infusion sites when potassium is added into the solution. It causes pain and increases the risk of infection, thus aggravating the emesis. The presence of potassium in hartman's solution made it superior to normal saline to correct hypokalaemia. Though it was noted that lower volume of hartman's solution was needed to correct hypokalaemia, it was not statistically significant (Table III). The significance might be different if the two types of fluid are compared in various degrees of hypokalaemia in patients. The amount of potassium supplement in Ringer Lactate i.e. $131 \mathrm{mmol} / \mathrm{L}$ may be not sufficient to correct hypokalaemia in severe cases

The fear of delayed clearance of acetonuria with the use of hartman's solution can be answered by the data in Table IV. It showed that there was no difference in the volume of either fluid to correct the starvation state indentified by the presence of acetonuria. There is a possibility that the lactate component in hartman's solution $(131 \mathrm{mmol} / \mathrm{L})$ could worsen the ketosis state of the patients. Surprisingly, despite the presence of acetonuria in all subjects, none of them had abnormal serum lactate level. This could be due to their early presentation to the hospital. This study did not demonstrate any elevation in the level of serum lactate in both pre- and post- treatment observations.

\section{CONCLUSION}

It could be concluded that both types of fluid (either normal saline or Ringer Lactate) could be used for fluid therapy in HG. Their efficacy in correcting dehydration was equally effective. Lactate component in Ringer lactate was not proven to worsen starvation among patients with HG. However, the efficacy of both fluids in treating various degree of hypokalaemia need to be further evaluated.

\section{REFERENCES}

1. Singer AJ, Brandt LJ. Pathophysiology of the gastrointestinal tract during pregnancy. Am J Gastro enterol. 1991; 86:1695-1712.

2. Mogadam M. Perspective as to pathogenesis and management of hyperemesis in pregnancy. Am J Gastroenterol. 1992; 87:806-7.

3. Newman V, Fullerton JT, Anderson PO. Clinical advances in the management of severe nausea and vomiting during pregnancy. JOGNN. 1993; 22:483-90.

4. Nelson- Piercy C. Treatment of nausea and vomiting in pregnancy. When should it be treated and what can be safely taken? Drug Saf. 1998;19(2):155-64
5. Philip B. Hyperemesis Gravidarum: Literature review. WMJ. 2003;102(3):46-51

6. Debra K. Gardner. Hyperemesis Gravidarum. @ http://www.uspharmacist.com

7. Anne-Marie Neill, Catherine Nelson-Piercy. "Hyperemesis gravidarum". The Obstetrician \& Gynaecologist; (2003) 3:204-207

8. Bashiri A, Neumann L, Maymon E, Katz M. "Hyperemesis gravidarum: epidemiologic features, complications and outcome”. Eur J Obstet Gynecol Reprod Biol. 1995; 63:135138

9. Depue RH, Bernstein L, Ross RK, Judd HL, Henderson BE. "Hyperemesis gravidarum in relation to estradiol levels, pregnancy out come and other maternal factors". Am J Obstet Gynecol. (1987)156:1137-1141

10. Jarnfelt-Samsioe A, Samsioe G, Velinder GM. "Nausea and vomiting in pregnancy-a contribution to its epidermiology". Gynecol Obstet Invest. (1983)16:221-229

11. SOGC Clinical Practice Guidelines. The management of nausea and vomiting in pregnancy. J Obstet Gynaecol Can. 2002;24(10):817-823

12. Fisher- Rasmussen W, Kjaer SK, Dahl C, Aspring U.Ginger treatment of hyperemesis gravidarum. Eur J Obstet Gynecol Reprod Biol. 1990;38:19-24

13. Seto A, Einarson T, Koren G. Pregnancy out come following first trimester exposure to anti- histamines: a meta- analysis. Am J Perinat. 1997;14(3):119-124

14. ACOG Practice Bulletin No. 52. Nausea and vomiting in pregnancy. 2004;103(4):803-815 
Table I. Basal parameters

\begin{tabular}{|lccc|} 
Parameter & $\begin{array}{c}\text { Hartman's } \\
\text { solution } \\
\text { group }\end{array}$ & $\begin{array}{c}\text { Normal } \\
\text { saline } \\
\text { group }\end{array}$ & P \\
\hline Age & $\begin{array}{c}25.22 \pm 6.06 \\
\text { years }\end{array}$ & $\begin{array}{c}25.36 \pm 4.58 \\
\text { years }\end{array}$ & NS \\
\hline
\end{tabular}

Table IV. Clearance of acetonuria

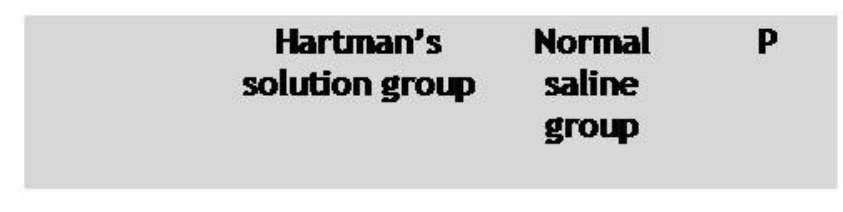

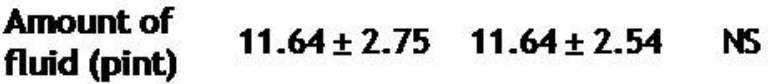 \\ fluid (pint)}

\begin{tabular}{|c|c|c|c|}
\hline Gravidity & $1.70 \pm 1.11$ & $2.34 \pm 1.57$ & NS \\
\hline $\begin{array}{l}\text { Gestational } \\
\text { age }\end{array}$ & $\begin{array}{c}10.70 \pm \\
3.21 \text { weeks }\end{array}$ & $\begin{array}{c}10.58 \pm 4.12 \\
\text { weeks }\end{array}$ & NS \\
\hline
\end{tabular}

$\begin{array}{lccc}\begin{array}{l}\text { Time taken } \\ \text { (hours) }\end{array} & 46.56 \pm 11.01 & 46.56 & \text { NS } \\ \text { (10.18 }\end{array}$

Note: Values are mean \pm SD unless otherwise noted.
BMI
$25.37 \pm, \quad 26.14 \pm$, NS
$2.49 \mathrm{~kg} / \mathrm{m}^{2} \quad 1.52 \mathrm{~kg} / \mathrm{m}^{2}$

$\mathrm{NS}=$ not significant

Table V. Lactate levels among hartman's solution group

\begin{tabular}{|c|c|c|c|}
\hline & $\begin{array}{c}\text { Pre- } \\
\text { treatment }\end{array}$ & $\begin{array}{l}\text { Post- } \\
\text { treatment }\end{array}$ & $\mathbf{P}$ \\
\hline $\begin{array}{l}\text { Lactate } \\
\text { level }\end{array}$ & $1.13 \pm 0.1 .90$ & $1.11 \pm 1.67$ & NS \\
\hline
\end{tabular}

Note: Values are mean \pm SD unless otherwise noted. $\mathrm{NS}=$ not significant

Note: Values are mean \pm SD unless otherwise noted. NS= not significant

Table III. Comparison of mean amount of fluid needed to correct hypokalaemia

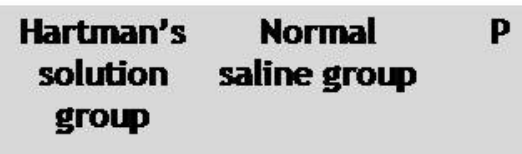

Amount of fluid (pint)
$8.34 \pm 2.44 \quad 8.88 \pm 2.63 \quad$ NS 